\title{
Further Studies of the Influence of a Ridge on the Low-Frequency Ground Wave
}

\author{
James R. Wait and Anabeth Murphy
}

\begin{abstract}
Computations are presented in graphical form for the perturbation of a plane wave by a semicylindrical boss on an otherwise flat ground plane of perfect conductivity. The height of the ridge is comparable to the wavelength. This is an extension of earlier work on the semielliptical boss.
\end{abstract}

A suggested model to study the effect of a nonsmooth ground for low frequencies is a semielliptical or semicylindrical boss on an otherwise flat ground plane of perfect conductivity. Using this model of a ridge, calculations were presented previously ${ }^{1}$ for a base width of twothirds of a wavelength and various ellipticity ratios. Recently, the computations for the semicylindrical case have been extended for other diameters. These results are presented here.

In the special case of the semicircular boss, the elliptic wave functions reduce to Bessel functions. The revelant solution is given in outline in the following.

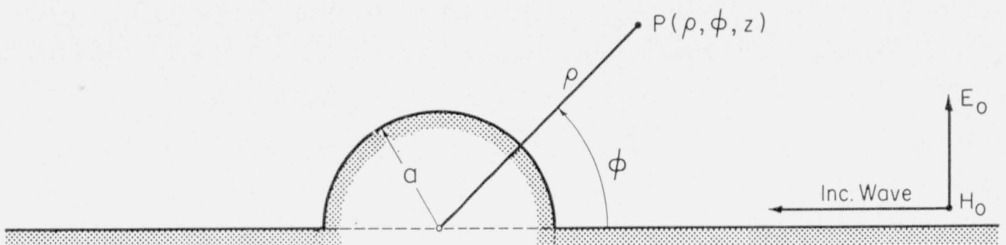

FIgure 1. Amplitude and phase of the field in the vicinity of a semicilindrical boss.

Semicylindrical boss on flat ground plane and incident plane wave.

The perfectly conducting semicylindrical boss of radius $a$ is illustrated in figure 1 , along with the cylindrical coordinate system $(\rho, \phi, z)$. The incident wave is incident from the right,

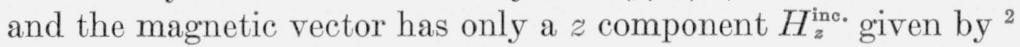

$$
H_{z}^{\mathrm{inco}}=H_{0} e^{i k_{\rho} \cos \phi},
$$

where $H_{0}$ is the amplitude of the magnetic field and $k=2 \pi$ /wavelength. The associated electric field is vertical and has an amplitude $E_{0}$. Using a well-known addition theorem in Bessel functions, ${ }^{3}$ the incident field can be rewritten in the form

$$
H_{z}^{\text {inc. }}=H_{0} \sum_{m=0}^{\infty} \epsilon_{m} e^{i m \pi / 2} J_{m}(k \rho) \cos m \phi,
$$

where

$$
\boldsymbol{\epsilon}_{0}=1, \quad \boldsymbol{\epsilon}_{m}=2(m=1,2,3 \ldots)
$$

The secondary, or scattered, field $H_{z}$ is now chosen to satisfy the boundary condition that the normal derivative of the total field is zero at $\rho=a$ for $\pi>\phi>0$ and at $\phi=0, \pi$ and $\rho>a$. Furthermore, the secondary field must contain the Hankel function $H_{m}^{(2)}(k \rho)$ to comply

\footnotetext{
1 J. R. Wait and A. Murphy, Influence of a ridge on the low-frequency ground wave, J. Research NBS 58, 1 (1957) RP2727.

2 The time factor implied is $\exp (i \omega t)$.

3 G. N. Watson, Theory of Bessel functions, Cambridge University Press, 2d ed. (1944).
} 
with the radiation condition at infinity. It then follows that

where

$$
H_{z}^{\text {see. }}=-H_{0} \sum_{m=0}^{\infty} \epsilon_{m} e^{i m \pi / 2} \frac{H_{m}^{(2)}(k \rho) J_{m}^{\prime}(x)}{H_{m}^{(2) \prime}(x)} \cos m \phi,
$$

$$
x=k a=2 \pi a / \lambda,
$$

and where the prime indicates a derivative of the Bessel function with respect to its argument.

The "relative amplitude" of the field is then defined by the quantity

$$
\left[\frac{H_{z}^{\text {sec. }}+H_{z}^{\text {inc. }}}{H_{0}}\right] \text {. }
$$

The "phase lag" of the field is defined by

$$
k \rho \cos \phi-\arg \left[\frac{H_{z}^{\text {sec. }}+H_{z}^{\text {inc. }}}{H_{0}}\right] .
$$

In other words, the relative amplitude is the magnitude of the total magnetic field for an incident wave of unit amplitude. The phase lag, as defined above, is the argument of the incident field less the argument of the total field.

The "relative amplitude" and "phase lag" for the magnetic field on the boss $\left(\rho=a, 180^{\circ}>\right.$ $\phi>0^{\circ}$ ) are plotted in figures 2 , a and $\mathrm{b}$, respectively, for various values of $x(=k a)$. The abscissa in both cases is the angular positon $\phi$ on the boss. It is immediately apparent that the amplitude of the field on the "illuminated" side $\left(\phi<90^{\circ}\right)$ of the boss is approaching 2.0 as the cylinder diameter is increasing. The amplitude of the field on the shadow side $\left(\phi>90^{\circ}\right)$ of the cylinder is strongly reduced for the larger cylinders. It is also interesting to note that the "phase lag" on the illuminated side is negative corresponding to a phase lead relative to the incident field while it tends to rise rather sharply on the shadow side. Geometrical optics would predict an amplitude of 2.0 and a zero phase for $0<\phi<90^{\circ}$ and a vanishing field for $90^{\circ}<\phi<180^{\circ}$. This is the asymptotic behavior as $x$ tends to infinity.

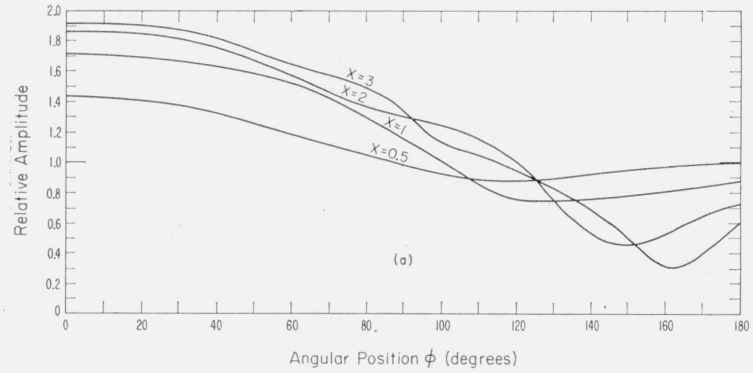

Figure 2. Amplitude and phase of the field in the vicinity of a semicylindrical boss.
(a) Amplitude of field on boss.
(b) Phase lag of field on boss,

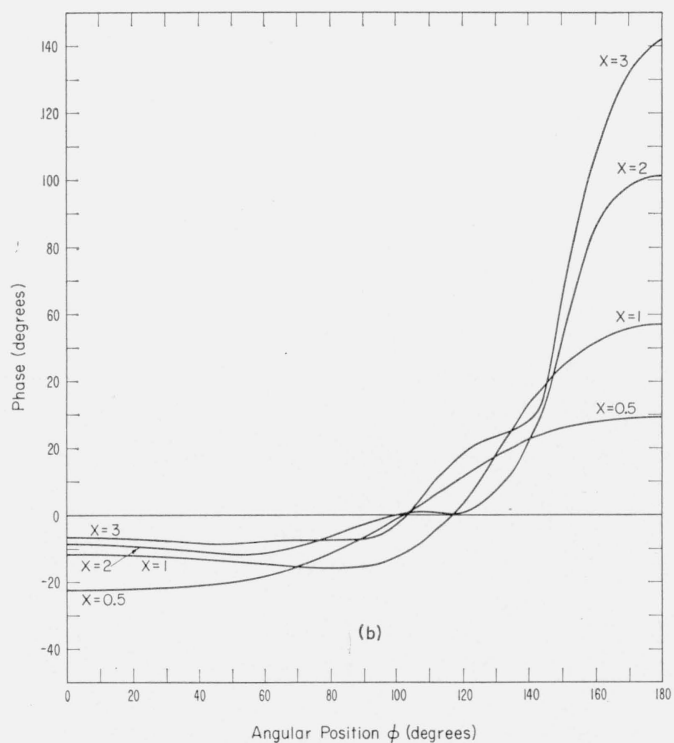

The "relative amplitude" and "phase" of the magnetic field on the ground plane in front of the boss $\left(\rho>a, \phi=0^{\circ}\right)$ are shown plotted in figures 3 , a and b, respectively, for a range of $x$. The abscissa is the distance measured from the center of the boss and expressed in radians. Here it is apparent that the secondary, or scattered, field is interfering with the incident wave to form a standing wave pattern. As would be expected, the period of the oscillation is approximately $\pi$ radians or one-half wavelength. 

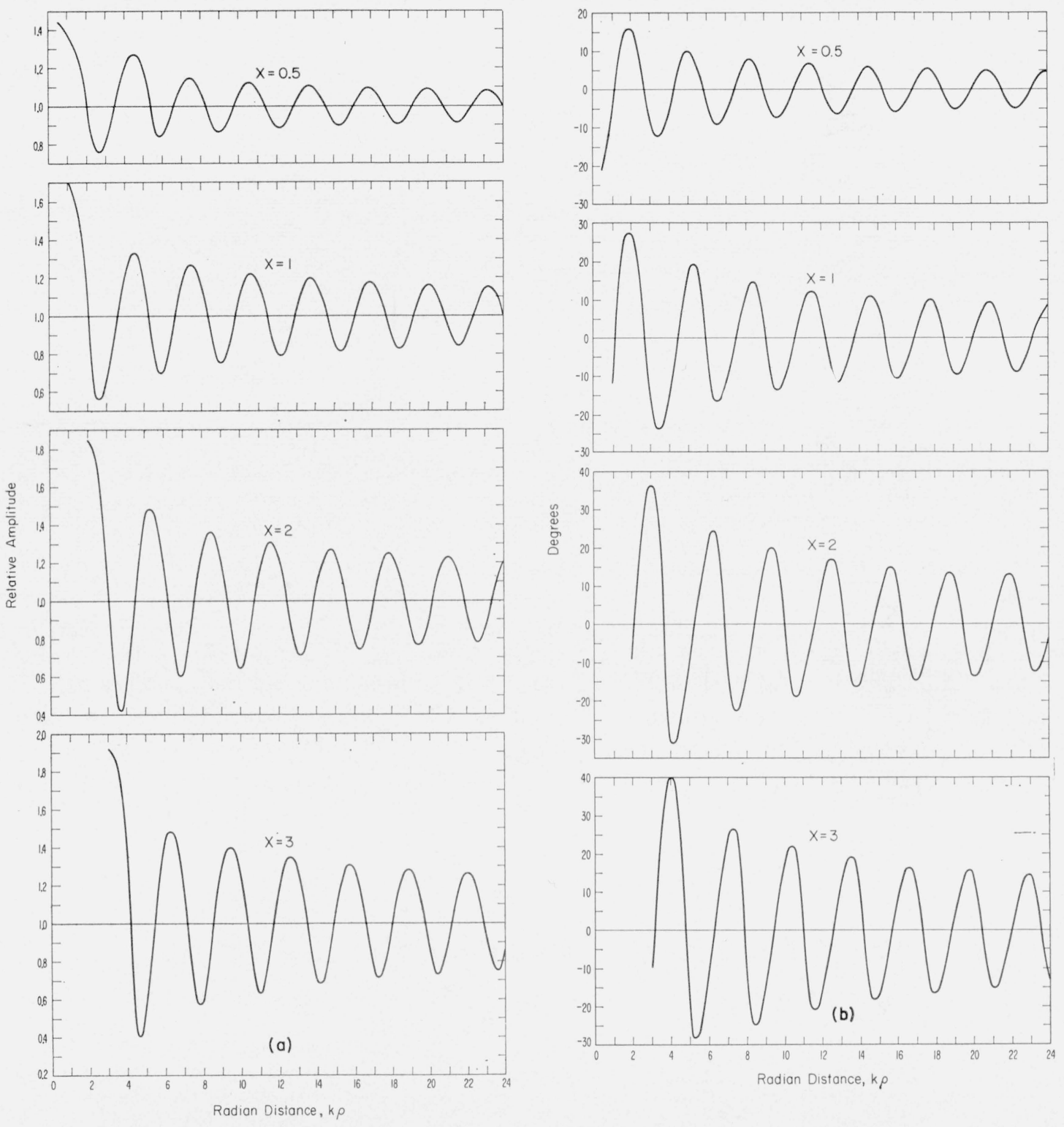

Figure 3. Amplitude and phase of the field in the vicinity of a semicylindrical boss.

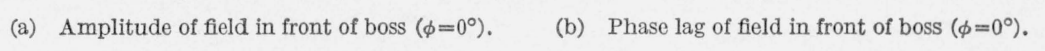

The same quantities for the ground plane to the rear of the boss $\left(\rho>a, \phi=180^{\circ}\right)$ are shown in figures 4 ,a and $\mathrm{b}$. It is seen that the relative amplitude of the field is approaching its unperturbed value of 1.0 as the observer recedes from the ridge and the phase lag is being restored to its unperturbed value of zero. As would be expected, the larger cylinders have a more pronounced influence.

The above model is admittedly highly idealized. The restriction, however, that the conductivity of the ridge is infinite can be easily removed in the formulation. The boundary condition on the cylinder is then to be replaced by

$$
E_{\phi} \cong-Z H_{z}
$$

where $Z$ is the surface impedance. ${ }^{4} Z$ for a good conductor, expressed as a ratio of the

4 M. A. Leontovich, Approximate boundary conditions for the electromagnetic field on the surface of a good conductor, Bul. Acad. Sci., U. S. S. R. serie physique, in Russian, 9, 16, (1944). 


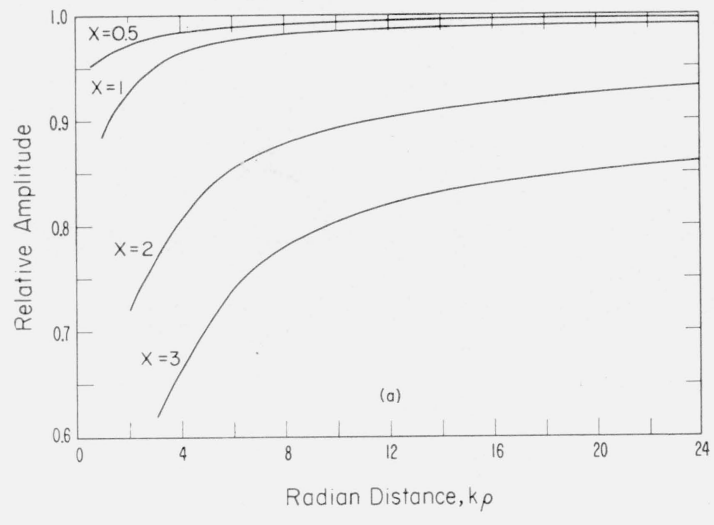

Figure 4. Amplitude and phase of the field in the vicinity of a semicylindrical boss.

(a) Amplitude of field to rear of boss $\left(\phi=180^{\circ}\right)$.

(b) Phase lag of field to rear of boss $\left(\phi=180^{\circ}\right)$.

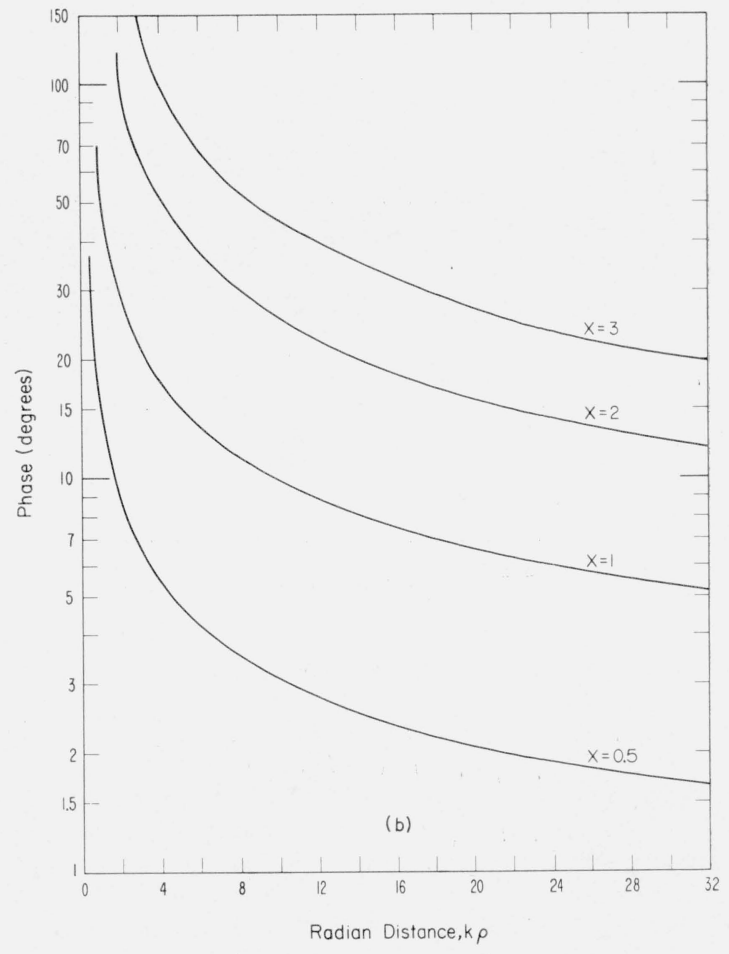

intrinsic impedance of free space, is given by

$$
Z / 120 \pi \cong(1+i)\left(\epsilon_{0} \omega / 2 \sigma\right)^{\frac{1}{2}} .
$$

$\epsilon_{0}$ is the dielectric constant of free space, and $\sigma$ is the conductivity of the cylindrical boss or ridge. Using this boundary condition, the secondary field becomes

where

$$
H_{z}^{\text {sec. }}=-H_{0} \sum_{m=0}^{\infty} \epsilon_{m} e^{i m \pi / 2} \frac{J_{m}^{\prime}(x)+G J_{m}(x)}{H_{m}^{(2) \prime}(x)+G H_{m}^{(2)}(x)} H_{m}^{(2)}(k \rho) \cos m \phi,
$$

$$
G=\frac{-i Z}{120 \pi} \cong(1-i)\left(\epsilon_{0} \omega / 2 \sigma\right)^{\frac{1}{2}}
$$

When $|G|<<1$ corresponding to a highly conducting cylinder, the above formula for $H_{z}^{\text {sec. }}$ becomes indistinguishable from its perfectly conducting counterpart given by eq (3).

The assumption of a perfectly conducting ground is not as simple to remove. However, if attention is confined to within several wavelengths of the ridge, the incident ground wave would behave locally as a plane wave at low-radio frequencies for a moderately or a wellconducting ground.

The restriction to normal incidence of the plane wave on the perfectly conducting cylindrical boss is easily removed. The only change necessary is to replace $k$ by $k \sin \theta$ everywhere ( $\theta$ is the angle subtended by the incident wave normal and the axis of the cylinder). The parameter $x$ in the figures is then equal to $k a \sin \theta$ and $k \rho$ is replaced by $k \rho \sin \theta$.

Ground-wave propagation over ridges or obstacles of finite length could be treated in the same fashion, using semispherical or semispheroidal bosses, although the computations would become excessively tedious. The results presented here for the semicylindrical boss and the earlier ones for the semielliptical boss are believed to be satisfactory to yield an initial estimate of the magnitude of the effect. Subsequent computations for more elaborate models would be justified if careful experimental measurements could be carried out under controlled conditions.

Boulder, Colo., March 13, 1958. 